\title{
Analytical approaches and preparation of biological, food and environmental samples for analyses of zearalenone and its metabolites
}

DOI: $10.1515 /$ revac-2020-0105

received May 21, 2020; accepted August 27, 2020

\begin{abstract}
Zearalenone (ZEN) is a mycotoxin that occurs in all stages of plant growth and development and exerts harmful effects on humans and animals. Zearalenone is easily absorbed in the digestive tract, and it is metabolized in the intestinal wall and the liver. Zearalenone has several derivatives: zearalenone, zearalanone (ZAN), $\alpha$-zearalenol ( $\alpha$-ZEL), $\beta$-zearalenol ( $\beta$-ZEL), $\alpha$-zearalanol $(\alpha-Z A L)$ and $\beta$-zearalanol ( $\beta$-ZAL). These substances have a high affinity for estrogen receptors, and they can gradually affect the endocrine system. Excess ZEN and its metabolites are excreted with urine and bile. This paper analyzes ZEN metabolism and investigates the presence of ZEN and its metabolites in urine. Since the isolation of ZEN and its metabolites from different matrices still poses a significant problem, the paper also presents various sample preparation methods (including liquid-solid extraction, liquid-liquid extraction and other techniques) as well as sensitive and specific chromatographic techniques, including liquid chromatography (LC) with fluorescence and mass spectrometry detection, gas chromatography (GC) and thin-layer chromatography (TLC).
\end{abstract}

Keywords: zearalenone, metabolites, urine, sample preparation, chromatographic analysis

\footnotetext{
*Corresponding author: Renata Gadzata-Kopciuch, Department of Environmental Chemistry and Bioanalytics, Faculty of Chemistry, Nicolaus Copernicus University in Toruń, Gagarina 7, 87-100 Toruń, Poland. Interdisciplinary Center for Modern Technologies, Nicolaus Copernicus University in Toruń, Wileńska 4, 87-100 Toruń, Poland. email: mailto:rgadz@umk.pl rgadz@umk.pl

Anna Kuźniewska, Bogustaw Buszewski: Department of Environmental Chemistry and Bioanalytics, Faculty of Chemistry, Nicolaus Copernicus University in Toruń, Gagarina 7, 87-100 Toruń, Poland. Interdisciplinary Center for Modern Technologies, Nicolaus Copernicus University in Toruń, Wileńska 4, 87-100 Toruń, Poland.
}

\section{Abbreviations}

ASE accelerated solvent extraction

DLLME dispersive liquid-liquid microextraction

DSPE dispersive solid phase extraction

GC-FID gas chromatography-flame ionization detection

HPLC high-performance liquid chromatography

IACs immunoaffinity columns

ILs ionic liquids

LOD limit of detection

LOQ limit of quantification

MAE microwave-assisted extraction

MSPE magnetic solid-phase extraction

PLE pressurized liquid extraction

SPE solid-phase extraction

RSD relative standard deviation

ZAN zearalanone

$\alpha$-ZAL $\alpha$-zearalanol

$\beta$-ZAL $\quad \beta$-zearalanol

$\alpha$-ZEL $\quad \alpha$-zearalenol

$\beta$-ZEL $\quad \beta$-zearalenol

ZEN zearalenone

\section{Introduction}

Mycotoxins are secondary metabolites produced by numerous fungi of the genera Aspergillus, Fusarium, and Penicillium. Mycotoxins may occur in food and feed as a result of fungal infections in crops. Due to their specific metabolism, the ingested mycotoxins are accumulated in different organs or tissues [1,2]. In 1960, the outbreak of the turkey X disease, which killed 100,000 ducks, turkeys and pheasants in the United Kingdom, led to a breakthrough in our understanding of mycotoxin-induced diseases. The affected animals were fed the same peanut mixture contaminated with a toxin produced mainly by Aspergillus flavus bacteria [2,3].

Zearalenone (ZEN) is a lactone of 6-[10-hydroxy-6oxy-trans-1-undecenyl]-B-resorcylic acid with a molecular weight of $318.364 \mathrm{~g} \mathrm{~mL}^{-1}$ [4-6]. Due to the presence of a 
double bond between $\mathrm{C} 11$ and $\mathrm{C} 12$, two stereoisomeric forms of ZEN are possible: trans-ZEN and cis-ZEN. The structure of ZEN is similar to that of natural estrogen, which enables the mycotoxin to gradually influence the hormonal system by binding to estrogen receptors [7]. As regards estrogen receptors, cis-ZEN is more similar to natural estrogen than trans-ZEN (Figure 1) [8,9]. The term "zearalenone" is a portmanteau combining zea for maize, ral for resorcylic acid lactone, en for the olefin double bond, and one for the ketone group. Zearalenone and its metabolites form $\alpha$ and $\beta$ derivatives [10].

Zearalenone has genotoxic, teratogenic, hemotoxic, immunotoxic, carcinogenic and hepatotoxic properties [11]. The maximum dose of ZEN is $50 \mu \mathrm{g} \mathrm{kg}^{-1}$ in cereals and maize-based snacks, $20 \mu \mathrm{g} \mathrm{kg}^{-1}$ in infant formulas and child products, and $200 \mu \mathrm{g} \mathrm{kg}^{-1}$ in unprocessed maize [12]. The maximum limit for direct human consumption is $100 \mu \mathrm{kg}^{-1}[13]$.

Zearalenone metabolites are formed via two major biotransformation pathways. The first pathway during which $\alpha$ - and $\beta$-zearalenol (ZEL) are formed involves hydroxylation catalyzed by $3 \alpha$ - and $3 \beta$-hydroxy-steroid dehydrogenases (HSDs). In the second pathway, ZEN and its metabolites are conjugated with glucuronic acid and catalyzed by uridine diphosphate glucuronyl transferases (UDPGT) [5,14]. Zearalenone and its metabolites are endocrine disruptors due to their structure and high affinity for estrogen receptors. Zearalenone undergoes metabolic changes, including reduction of the main $\alpha$ - and $\beta$-metabolites of zearalenol and conjugation of ZEN and its metabolites with glucuronic or sulfuric acid (Figure 2). The above processes increase the polarity of ZEN molecules and influence the elimination of toxic substances from the body [15]. Excess ZEN and its metabolites are excreted from the body with urine and bile. Various methods for enriching and isolating toxic compounds from biological matrices such as blood, tissues or urine have been developed $[16,17]$.
The isolation of analytes from different matrices, mainly biological and environmental samples, and the purification and enrichment of extracts pose considerable problems in analytical practice. The most common methods of isolating ZEN and its metabolites include solidphase extraction with sorbents such as octadecyl $[18,19]$ as well as immunosorbents [20] and molecularly imprinted polymers [21]. Pressurized liquid extraction (PLE) [22] and accelerated solvent extraction (ASE) [4] are also excellent methods for isolating the analyte. Various chemical and biochemical methods are used to quantify ZEN and its metabolites. Zearalenone concentrations in biological and environmental samples are generally very low.

Different quantitative indicators are used to determine the concentrations of ZEN and its metabolites. Instrumental analyses should be characterized by high sensitivity and a low limit of detection and quantification. Trace amounts of ZEN in extracts are determined by liquid chromatography with different detection methods and gas chromatography.

The aim of this study was to describe different analytical approaches in preparation of biological, food and environmental samples for analyses of zearalenone and its metabolites. Special attention was also paid to the assessment of the exposure of living organisms to the influence of ZEN and the appearance of its metabolites in urine.

\section{Urine as a biological sample for assessing the effects of exposure to ZEN}

There is a general scarcity of research on the toxic and harmful effects of ZEN compounds in humans. Most studies have been conducted on animal samples which are also important for evaluating the health risks associated with ZEN exposure in humans. In both humans and animals, ZEN and

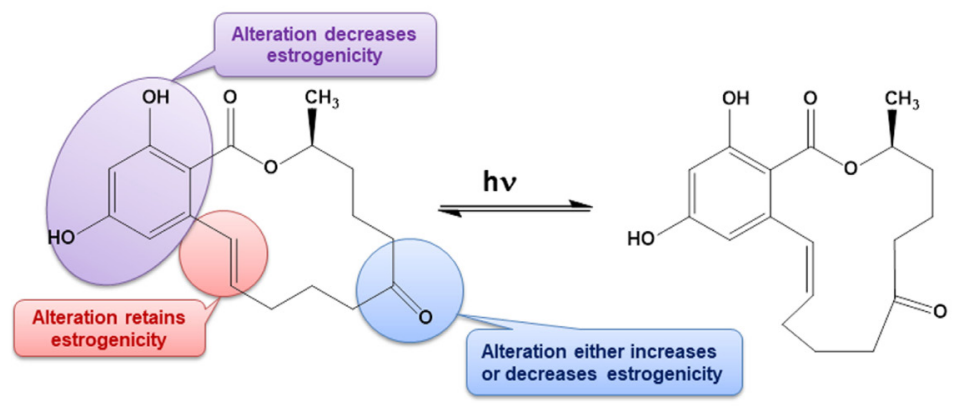

Figure 1: Structure of cis (a) and trans (b) zearalenone (ZEN). 


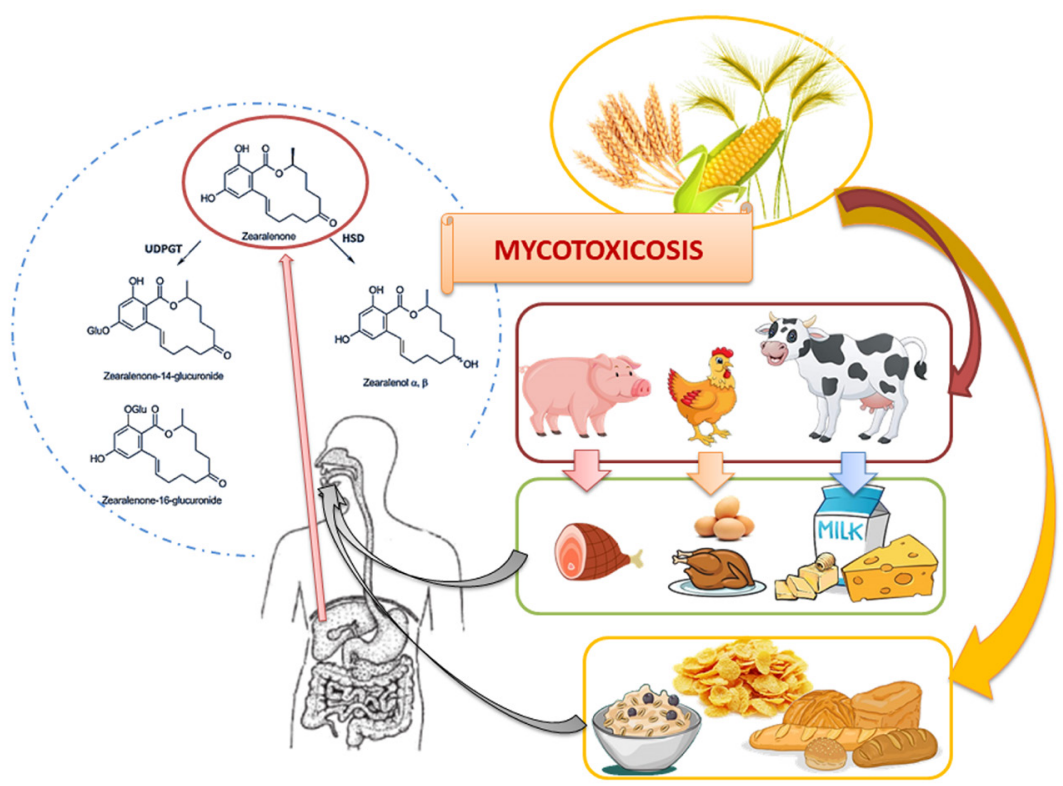

Figure 2: The infection pathway and zearalenone metabolism.

its metabolites are excreted mainly with urine. The research related to animals' use has been complied with all the relevant national regulations and institutional policies for the care and use of animals. Similarly, in the case of research to human use has been complied with all the relevant national regulations, institutional policies and in accordance the tenets of the Helsinki Declaration, and has been approved by the authors' institutional review board or equivalent committee.

The biological recovery of ZEN from porcine urine depends on the route of administration. Urine recovery ranges from $14 \%$ to $45.38 \%$ of the applied dose after oral administration, and from $45.4 \%$ to $77 \%$ after intravenous injection [23,24]. In a study by Dänicke et al. [25,26], piglets were fed a diet with increasing inclusion levels of wheat contaminated with Fusarium toxin over a period of 35 days. The toxin was identified by hydrolysis with $\beta$-glucuronidase/sulfatase, immunoaffinity clean-up and high-performance liquid chromatography (HPLC) with fluorescence detection. A linear dose relationship was observed with an increase in ZEN, $\alpha$-ZEL and $\beta$-ZEL concentration in urine. The average urine recovery for this mycotoxin was $94.75 \%$ and $85 \%$, respectively.

In the work of Gambacorta et al. [24], piglets were administered a single dietary bolus of mixtures of deoxynivalenol (DON), aflatoxin $\mathrm{B}_{1}\left(\mathrm{AFB}_{1}\right)$, fumonisin $\mathrm{B}_{1}$ $\left(\mathrm{FB}_{1}\right)$, ochratoxin $\mathrm{A}(\mathrm{OTA})$ and $\mathrm{ZEN}$. Urine samples were collected three times over a 24-hour period. The samples were hydrolyzed with $\beta$-glucuronidase/sulfatase, purified on an immunoaffinity column (IAC; Myco6in1, Vicam) and Oasis ${ }^{\circledR}$ HLB solid phase extraction. Mycotoxin biomarkers were detected in each urine sample, and $\beta$-ZEL was identified only at the highest dose of ZEN. The percentage of the mycotoxin excreted in $24 \mathrm{~h}$ was calculated based on the volume and concentration of urine in the analyte. The mean percentage of ZEN excreted in urine during $24 \mathrm{~h}$ was $36.8 \%$ (28.4\% and $8.3 \%$ in a 3:1 ratio of ZEN/ $\alpha-Z E L)$.

Warth et al. [27] monitored a 27-year-old male patient for the content of ZEN excreted in urine. The patient was administered a naturally contaminated diet for four days. After enzymatic hydrolysis, total ZEN concentration was measured in 24-hour urine samples. The total ZEN content (mainly in the form of glucuronide) ranged from 0.30 to $0.59 \mathrm{ng} \mathrm{mL}{ }^{-1}$, which corresponded to $7.0-13.2 \%$ of the administered daily dose. It should also be noted that $\alpha$ and $\beta$-ZEL were not identified in excreted urine

A study performed on 30 men and 30 women in Germany [28] revealed the presence of ZEN, $\alpha$-ZEL and $\beta$-ZEL in all urine samples. The mycotoxin and its metabolites were identified by LC-MS/MS after enzymatic hydrolysis and were purified on the immunoaffinity column. The concentration of ZEN ranged from 0.04 to $0.28 \mathrm{ng} \mathrm{mL}^{-1}$, the content of $\alpha$-ZEL - from 0.06 to $0.45 \mathrm{ng} \mathrm{mL}^{-1}$, and $\beta$-ZEL - from 0.01 to $0.20 \mathrm{ng} \mathrm{mL}^{-1}$. Significant gender-related differences were not reported in the composition and concentrations of the analytes in urine. The values noted in the majority of the exposed individuals were below the tolerable daily intake level of $0.25 \mu \mathrm{g} \mathrm{kg}^{-1} \mathrm{BW}$ set by EFSA, but higher values were observed in some subjects.

The urinary excretion of ZEN appears to be a reliable indicator of human exposure to this mycotoxin. The 
concentrations of ZEN and its metabolites, including $\alpha$ and $\beta$-ZEL, provide valuable information about exposure to modified ZEN mycotoxins which co-occur in food and contribute to human exposure. The health risks associated with the exposure to ZEN have been researched extensively in animals (mainly pigs), but far less frequently in humans. Research has revealed that the formation and distribution of ZEN metabolites are similar in humans and animals. Despite the fact that pigs were administered a higher dose than humans, the proportions of reduced forms of ZEN in urine were comparable. These findings considerably expand our understanding of ZEN's toxicity.

\section{Mycotoxin isolation methods}

The sample preparation process exerts the greatest influence on the results of quantitative analyses. Different sample preparation procedures (Figure 3) are used to optimize the efficiency of the analytical procedure.

\subsection{Solid-phase extraction}

During solid-phase extraction, a solution of the analyte is passed through an extraction column containing the adsorbent. The analytes are absorbed by the particle bed, and the column is washed to remove the impurities. In the next step, the absorbed analyte is washed out with a suitable solvent. Numerous examples of solid-phase extraction with different sorbents (Table 1), including silica gel modified by the octadecyl hydrocarbon chain (C18) [19,20,34], Florisil [19] and Oasis ${ }^{\circledR}$ HLB polymers [24], as well as extract purification tools such as immunosorbents (IAC) $[20,35,36]$ can be found in the literature.

Jodlbauer et al. [34] used the octadecyl sorbent to isolate ZEN and its derivatives from urine and animal tissue samples. The recovery of ZEN and its metabolites from urine and tissues ranged from $86 \%$ to $102 \%$ in the evaluated method. Andres et al. [19] used C18 and Florisil as sorbents in the process of ZEN isolation and purification. Both sorbents were tested on urine samples, and Florisil was found to be less selective. The octadecyl sorbent was selected for further analyses, where ZEN recovery ranged from $92 \%$ to $108 \%$.

In other studies, ZEN and its metabolites were isolated from urine, plasma and equine feces, and were purified by solid-phase extraction (SPE) and immunoaffinity chromatography (IAC) [20]. An ammonium acetate buffer with $\mathrm{pH} 4.8$ and $\mathrm{D}_{2}$-ZAN was initially added to the urine sample. The solution was incubated with $\beta$-glucuronidase/ arylsulfhatase solution and brought to $\mathrm{pH}$ 4. The solution was applied to a sorbent filled with ISOLUTE ${ }^{\circledR}$ C18, which was previously conditioned with methanol and $\mathrm{H}_{2} \mathrm{O}$. The sorbent was washed with $30 \% \mathrm{MeOH}(2 \mathrm{~mL})$ and dried under vacuum. The analytes were eluted with methanol $(1.25 \mathrm{~mL})$, and the eluate was diluted with a phosphate buffer solution at $\mathrm{pH}$ 7.4. The extract was purified with an immunosorbent (Easi-Extract ${ }^{\circledR}$ Zearalenone) which was previously conditioned with phosphate buffered saline solution (PBS). The cited study demonstrated that effective sample preparation and sensitivity of the method are determined

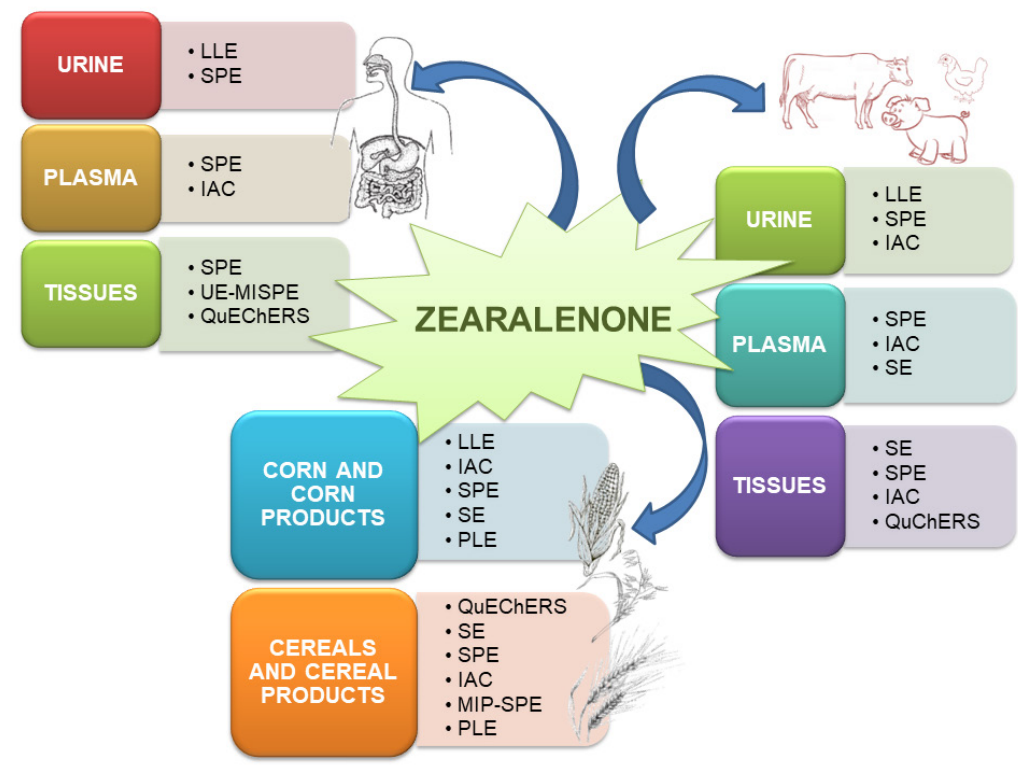

Figure 3: Various extraction techniques for isolation of zearalenone and its metabolites. 
Table 1: Solid-phase extraction with different sorbents and a comparison of recovery values

\begin{tabular}{|c|c|c|c|c|}
\hline Mycotoxin & Matrix & Sample preparation & Recovery & References \\
\hline $\begin{array}{l}\text { ZEN } \\
\alpha-Z E L\end{array}$ & Endometrial cancer & UE-MISPE & $82.5-97.9 \%$ & {$[16]$} \\
\hline $\begin{array}{l}\text { ZEN } \\
\alpha-Z A L \\
\beta-Z A L \\
\alpha-Z E L \\
\beta-Z E L \\
\text { ZAN }\end{array}$ & $\begin{array}{l}\text { Human, bovine and swine } \\
\text { urine }\end{array}$ & C18/Florisil & $87-108 \%$ & [19] \\
\hline $\begin{array}{l}\text { ZEN } \\
\alpha-Z E L \\
\beta-Z E L\end{array}$ & Urine & IACs (Myco6in1, VICAM) & $\mathrm{nd}^{*}$ & {$[23]$} \\
\hline $\begin{array}{l}\text { ZEN } \\
\alpha-Z E L \\
\beta-Z E L\end{array}$ & $\begin{array}{l}\text { Serum } \\
\text { Feedstuffs } \\
\text { Bile } \\
\text { Urine } \\
\text { Liver } \\
\end{array}$ & IACs (Easi-Extract ${ }^{\mathrm{TM}}$ ZON, R-Biopharm) & $\begin{array}{l}66-88 \% \\
74-82 \% \\
81-104 \% \\
75-94 \% \\
66-88 \%\end{array}$ & {$[25,26]$} \\
\hline $\begin{array}{l}\text { ZEN } \\
\alpha-Z E L \\
\beta-Z E L\end{array}$ & Urine & IACs (ZearalaTest $\left.{ }^{\mathrm{TM}}, \mathrm{VICAM}\right)$ & $\mathrm{nd}^{*}$ & {$[28]$} \\
\hline $\begin{array}{l}\text { ZEN } \\
\alpha-Z A L \\
\beta-Z A L\end{array}$ & $\begin{array}{l}\text { Bovine feed } \\
\text { Urine }\end{array}$ & SPE (C18) & $\begin{array}{l}79 \% \\
82 \%\end{array}$ & {$[29]$} \\
\hline ZEN & Rainbow trout organs & IACs (ZearalaTest ${ }^{\text {TMWB, VICAM) }}$ & $85 \%$ & [30] \\
\hline ZEN & Functional food & QuEChERS (DSPE) & $83-91.5 \%$ & [31] \\
\hline ZEN & Corn and corn products & $\mathrm{IACs}$ & $92-114 \%$ & {$[33]$} \\
\hline ZEN & $\begin{array}{l}\text { Urine } \\
\text { Animal tissue }\end{array}$ & SPE RP-C18 (Phenomenex) & $86-102 \%$ & {$[34]$} \\
\hline ZEN & Blood & IACs (ZearalaTest $\left.{ }^{\mathrm{TM}}, \mathrm{VICAM}\right)$ & $\mathrm{nd}^{*}$ & {$[35]$} \\
\hline
\end{tabular}

*) nd - no data

by the complexity of the analyzed matrix. Single-stage purification before IAC was sufficient for plasma samples, whereas urine samples had to be purified by both SPE and IAC. Urine recovery ranged from $56 \%$ to $100 \%$.

Zearalenone and its metabolites can also be isolated with molecularly imprinted polymers (MIP). In the work of Mausia et al. [21], the zearalenone template was synthesized by precipitation polymerisation and applied as a sorbent in molecularly imprinted solid-phase extraction (MISPE). The sorbent was characterized by high stability and affinity for the ZEN molecule. The sorbent was previously conditioned with acetonitrile; the sample was applied in an acetonitrile/ water mixture $(60 / 40 \% \mathrm{v} / \mathrm{v})$ and washed with an ACN/ $\mathrm{H}_{2} \mathrm{O}$ mixture $(30 / 70 \% \mathrm{v} / \mathrm{v})$. The analytes were eluted with a $95 / 5 \%(\mathrm{v} / \mathrm{v})$ mixture of methanol and acetic acid. The obtained recovery for ZEN ranged from $90.8 \%$ to $99.6 \%$.

Comments: Since the introduction of solid phase extraction as a sample preparation method (especially for liquid samples), this technique has been widely used to isolate and enrich various compounds and has often been used to purify extracts from interfering compounds. The rapid development of SPE is due to progress aimed at the synthesis of new sorbents that are characterized by specific interactions with an isolated compound in order to achieve high recovery. Future research should focus on reducing the amount of sorbent used for extraction, thus minimizing or eliminating the use of toxic solvents. An alternative to traditional sorbents can be molecularly imprinted polymers (MIPs), which enable selective and specific interaction with the analyte. This ensures not only selective retention of the isolated compound, but also purification of the extract from interfering compounds. Undoubtedly, an important issue is the repeatability and reproducibility of the obtained sorbents.

\subsection{QuEChERS}

The quick, easy, cheap, effective, rugged and safe (QuEChERS) method is suitable for preparing biological 
samples in line with green chemistry rules. In the QuEChERS approach, the isolated compound is extracted with organic solvents with the addition of salt, and analyte and matrix components are split between the organic phase and water. A purified extract is obtained by dispersion and solid phase extraction. The QuEChERS method is simple, it relies on non-chlorinated solvents, involves fewer sample preparation steps, and can be used to analyze a larger number of samples. Pajewska at el. [17] and Woźniak at el. [37] used the QuEChERS technique to isolate ZEN and its metabolites from muscle tissues, and obtained a recovery rate of $56-115 \%$. This technique was also used by Cunha and Fernandes [38] to analyze breakfast cereals and contaminated flour. The procedure was modified to produce a recovery rate of $67-101 \%$ for breakfast cereals and 52-103\% for flour.

\subsection{Novel methods of sample preparation for the isolation of ZEN and its metabolites}

Zearalenone levels should be monitored in food and feed to protect humans and animals from consuming products contaminated with this mycotoxin. Therefore, innovative methods are being sought to minimize the health problems associated with toxins.

Kim et al. [39] developed an innovative system for removing and monitoring ZEN levels in feed, which offers an alternative to IAC. The proposed approach relies on the use of monoclonal antibodies (mAbs) and magnetic nanoparticles (MNP). In the cited study, ground and enriched samples were shaken and extracted in $70 \%$ methanol. The collected samples were passed through paper filters, and MNP-mAb conjugates were used to remove the mycotoxin. Zearalenone was removed magnetically from the supernatant, and its concentration was measured. The mycotoxin was also extracted by IAC for comparative purposes. The recovery rate was significantly higher in the proposed approach (92-100\%) than in IAC (81-88\%).

Amoli-Diva et al. [40] used dispersive liquid-liquid micro-extraction (DLLME) combined with microsolid phase extraction ( $\mu$-SPE) to determine ZEN levels in wheat samples. This simple and low-cost extraction method was characterized by satisfactory repeatability and reproducibility as well as high recovery in the range of $91.6 \%$ to $99.1 \%$. Antep and Merdivan [41] investigated the effectiveness of the DLLME method for quantitative determination of ZEN in beer, with the aim of developing a simple, fast and sensitive extraction method. The obtained recoveries ranged from $71 \%$ to
$108 \%$. The simplicity, accuracy and low cost of the DLLME- $\mu$-SPE method were confirmed by Hashemi et al. [42] who extracted ZEN from an acetonitrile mixture with the use of hydrophobic magnetic nanoparticles. The magnetic absorber was collected with an external magnet, and water from the supernatant was decanted. The recovery rate for maize samples was high in the range of $93.2 \%$ to $102.1 \%$. The study demonstrated that ZEN levels in samples could be effectively determined with the use of the two-stage micro-extraction procedure based on DLLME and $\mu$-SPE with hydrophobic magnetic nanoparticles.

Porto-Figueira et al. [43] combined $\mu$-QuEChERS with ultrasound-assisted extraction (USAE) to improve the original QuEChERS method. The described approach involved a reduced amount of separating salts, smaller samples and fewer solvents. The cited authors demonstrated the potential of the proposed method.

Magnetic molecularly imprinted polymers (mMIP) were used for selective extraction of ZEN from various cereal flours [44]. Quercetin was the inert template, and it was also used to synthesize mMIP for ZEN. Flour extraction mixtures were prepared by centrifugation and sonication with acetonitrile and water $(80: 20, \mathrm{v} / \mathrm{v})$ and $0.2 \% \mathrm{HCOOH}$. The extracts were centrifuged, separated and placed in centrifuge tubes, and ultra-pure water and $100 \mathrm{mg}$ of mMIP were added. The solution was decanted, and the supernatant was removed with an external magnet. The analyte with MMIP was transferred to a water test tube, and the supernatant was centrifuged and removed. Zearalenone was eluted three times with methanol and twice with acetonitrile. The sample was centrifuged each time, and mMIP was magnetically decanted. The ZEN recovery rate was above $95 \%$, which indicates that this method is useful for isolating trace amounts of the mycotoxin in food analyses.

Another unconventional method for ZEN detection was presented by Fang et al. [45]. The authors synthesized a new molecularly imprinted optosensing material (MIOM) by anchoring the MIP layer on ionic liquid-modified CdSe/ ZnS quantum dots. The MIOM was used to detect ZEN in maize, rice and wheat flours. The recovery rates ranged from $84 \%$ to $107 \%$.

Comments: The novel approaches to sample preparation differ from conventional methods. The separation of the extracts from other co-extracted compounds in the matrix is also an important issue during sample preparation. Effective sample preparation and high sensitivity of the method are determined by the complexity of the matrix, therefore each step is an inherent element to obtaining the best 
possible recovery results. It is preferable to replace the expensive sorbents (e.g., antibodies) by magnetic particle imprinted polymers with structural analogs of the analytes. The developed methodologies for isolating ZEN and its metabolites with the use of MMIP allow obtaining high and not always repeatable recoveries, but they allow high efficiency of cleaning the samples from compounds that coexist in them as contaminants. Future research should focus on the search for a template for the molecular imprint and the development of a repeatable synthesis of the prepared polymers. In addition, new applications such as integrated artificial intelligence systems to predict appropriate extraction conditions are expected to ensure the future viability of this technique.

\subsection{Solvent extraction}

Liquid extraction is a popular method of extracting analytes from the matrix, including samples of food and infected animal tissues, in analyses of ZEN and its metabolites. Additional ultrasonic (UE) or microwave (MEA) energy can be used in this extraction technique, which can successfully increase the efficiency of extraction and shorten the time of its operation.

Pallaroni et al. [22] described the pressurized liquid extraction (PLE) method that relies on environmentally acceptable and less harmful organic solvents. Zearalenone was extracted from maize with a 50/50\% (v/v) mixture of isopropanol and an aqueous solution of triethanolamine $(1 \%)$ at $80^{\circ} \mathrm{C}$. The obtained recovery was $85 \%$.

Uracca et al. [4] determined the content of ZEN and $\alpha$-zearalenone in cereal samples (corn, wheat, rice, barley, rye) and swine feed by ASE. Optimal extraction conditions were obtained by using a solvent mixture $\mathrm{MeOH} / \mathrm{ACN}$ $(50: 50 \mathrm{v} / \mathrm{v})$ at the temperature of $50^{\circ} \mathrm{C}$, a pressure of $1500 \mathrm{psi}$, a static time of $5 \mathrm{~min}$ and flush volume $60 \%$. The obtained recovery exceeded 96\% for ZEN (RSD < 4.0\%) and reached $98 \%$ for $\alpha-Z A L$ (RSD < 4.6\%) [4].

Comments: One of the advantages of ASE method is that the process is automated. The solvent thoroughly penetrates the sample, which reduces extraction time and decreases cost associated with slow sample preparation.

\section{Analytical methods}

Various chemical and biochemical methods are used to quantify ZEN and its metabolites and minimize the health risks associated with exposure to mycotoxins.
Biological and environmental samples contain trace amounts of these mycotoxins, which is why selective and sensitive identification techniques with low limits of detection (LOD) and determination (LOQ) are required. Various analytical techniques and the relevant LOD values for ZEN and its metabolites in different matrices are presented in Table 2 .

\subsection{High-performance liquid chromatography}

The presence of ZEN and its metabolites can be determined by high-performance liquid chromatography (HPLC) with various detectors. Zearalenone is a naturally fluorescent compound, and it is identified by fluorimetric detection.

Ok et al. [36] isolated ZEN by HPLC with fluorimetric detection, where the Synergi ${ }^{\mathrm{TM}}$ Hydro-RP $80 \AA$ column (Phenomenex, $4.6 \times 250 \mathrm{~mm} ; 4 \mu \mathrm{m}$ ) was maintained at $40^{\circ} \mathrm{C}$. The excitation and emission wavelengths were $275 \mathrm{~nm}$ and $450 \mathrm{~nm}$, respectively. $\mathrm{A}_{2} \mathrm{O} / \mathrm{ACN} / \mathrm{MeOH}$ $(35 / 10 / 55 \% \mathrm{v} / \mathrm{v} / \mathrm{v})$ mixture was the mobile phase, and the flow rate was $1.0 \mathrm{~mL} \mathrm{~min}^{-1}$. The LOD value was $4.0 \mu \mathrm{g} \mathrm{kg}^{-1}$, and LOQ was determined at $10.0 \mu \mathrm{gg}^{-1}$.

Marczuk et al. [35] analyzed clinical cases of dairy cattle infected by Fusarium fungi, including ZEN. Blood samples were collected and subjected to IAC extraction with a Zearala-Test ${ }^{\circledR}$ column to determine the plasma concentration of ZEN. The extracts were analyzed by HPLC with fluorescent detection. An ODS Hypersil chromatographic column $(4 \times 250 \mathrm{~mm} ; 5 \mu \mathrm{m})$ was used. The mobile phase was an $\mathrm{ACN} / \mathrm{MeOH} / \mathrm{H}_{2} \mathrm{O}$ mixture $(46 / 8 / 46$ or $8 / 10 / 82 \% \mathrm{v} / \mathrm{v} / \mathrm{v})$ with a flow rate of $1.8 \mathrm{~mL}$ $\mathrm{min}^{-1}$ or $1.5 \mathrm{~mL} \mathrm{~min}^{-1}$ at $30^{\circ} \mathrm{C}$. The excitation and emission wavelengths were 218 and $438 \mathrm{~nm}$, respectively.

Hewitt et al. [33] identified ZEN in corn and maize products with the use of an $\mathrm{H}_{2} \mathrm{O} / \mathrm{MeOH} / \mathrm{ACN}$ mixture as the mobile phase at an excitation wavelength of $274 \mathrm{~nm}$ and an emission wavelength of $440 \mathrm{~nm}$. Pajewska et al. [17] relied on the QuEChERS technique to isolate ZEN and its metabolites from human tissues. The compounds were separated by HPLC with fluorimetric detection at the excitation and emission wavelengths of 270 and $440 \mathrm{~nm}$, respectively. Zearalenone and $\alpha$-ZEL were detected as compounds with the highest fluorescent intensity. Highperformance liquid chromatography with fluorescence detection is characterized by high selectivity and sensitivity, and it is one of the most popular methods of ZEN detection.

Liquid chromatography coupled with mass spectrometry is becoming an increasingly popular 
Table 2: Various analytical techniques for detecting ZEN and its metabolites in different matrices

\begin{tabular}{|c|c|c|c|c|c|}
\hline Mycotoxin & Matrix & Sample preparation & Technique & LOD & References \\
\hline $\begin{array}{l}\text { ZEN } \\
\alpha-Z A L \\
\beta-Z A L \\
\alpha-Z E L \\
\beta-Z E L Z A N\end{array}$ & broiler meat & SE, IACS & HPLC-DAD & $30-140 \mathrm{ng} \mathrm{mL}^{-1}$ & {$[52]$} \\
\hline ZEN & $\begin{array}{l}\text { maize and cereal-based } \\
\text { feeds }\end{array}$ & SPE C18 & HPLC-FLD & $15 \mathrm{ng} \mathrm{mL}^{-1}$ & {$[53]$} \\
\hline ZEN & plasma & IACs & HPLC-FLD & $5.94-14.30 \mathrm{ng} \mathrm{mL}^{-1}$ & {$[35]$} \\
\hline $\begin{array}{l}\text { ZEN } \\
\text { ZAN }\end{array}$ & grain & SPE C18, IACs & HPLC-MS/MS & $0.5 \mathrm{ng} \mathrm{g}^{-1}$ & {$[18]$} \\
\hline $\begin{array}{l}\text { ZEN } \\
\alpha-Z E L \\
\beta-Z E L\end{array}$ & cereal-based foods & SPE, IACS & HPLC-MS/MS & $1-10 \mathrm{ng} \mathrm{g}^{-1}$ & {$[54]$} \\
\hline $\begin{array}{l}\text { ZEN } \\
\alpha \text {-ZAL } \\
\beta \text {-ZAL } \\
\alpha \text {-ZEL } \\
\beta \text {-ZEL ZAN }\end{array}$ & sediments & PLE, SPE & GC-MS & $0.01-10.28 \mathrm{ng} \mathrm{g}^{-1}$ & {$[55]$} \\
\hline $\begin{array}{l}\text { ZEN } \\
\alpha-Z E L \\
\beta-Z E L\end{array}$ & biological samples & SE, SPE & GC-MS & $0.06-0.6 \mathrm{ng} \mathrm{mL}^{-1}$ & {$[56]$} \\
\hline ZEN & maize & SE, SPE C18 & HPLC, TLC & $20 \mathrm{ng} \mathrm{g}^{-1}$ & {$[57]$} \\
\hline
\end{tabular}

detection technique despite its high cost. Liquid chromatography with one mass analyzer (LC-MS) and tandem mass spectrometry (LC-MS/MS) ensure unambiguous identification of the analyte. This method requires an ion source to determine the mass-to-charge ratio $(\mathrm{m} / \mathrm{z})$. Electrospray ionization (ESI) and atmospheric pressure chemical ionization (APCI) are the most widely used ionization techniques $[46,47]$.

Jodlbauer et al. [34] relied on the LC-MS/MS technique with an APCI type ionization source to determine the concentrations of ZEN and its metabolites in urine and tissue samples collected from cows and pigs. The analysis was performed on the Supersphere ${ }^{\circledR} \mathrm{RP}-18$ Merck column $(125 \times 3.0 \mathrm{~mm}$; $4 \mu \mathrm{m})$ at $35^{\circ} \mathrm{C}$. The mobile phase was an $\mathrm{ACN} / \mathrm{MeOH} /$ $\mathrm{H}_{2} \mathrm{O}(10 / 45 / 45 \% \mathrm{v} / \mathrm{v} / \mathrm{v})$ mixture with $15 \mathrm{mM}$ ammonium acetate at a flow rate of $0.5 \mathrm{~mL} \mathrm{~min}^{-1}$. The LOD values ranged from 0.1 to $0.5 \mu \mathrm{g} \mathrm{L}^{-1}$, and $\mathrm{LOQ}$ - from 0.5 to $1 \mu \mathrm{g} \mathrm{L}^{-1}$.

Soleimanes et al. [47] determined ZEN levels in cereal matrices by the UPLC-MS/MS technique with ESI in positive and negative ion mode. The LOD values in the tested matrices were determined at 0.1-0.4 $\mathrm{ng} \mathrm{g}^{-1}$, and LOQ values - at 0.2-1 $\mathrm{ng} \mathrm{g}^{-1}$. Liquid chromatography coupled with mass spectrometry is increasingly used in analyses, but other analytical methods are often selected due to the high cost of mass spectrometry.

\subsection{Gas chromatography}

Gas chromatography-mass spectrometry (GC-MS) is also used to analyze ZEN and its metabolites. A GC-MS method for detecting ZEN and its metabolites in samples of bovine urine, bile, meat and kidneys was described by the Standard Operating Procedure ARO/458 [48]. The GC-MS/MS method was also used by Rodríguez-Carrasco et al. [49] to detect ZEN in grain-based products. Dudziak [50] relied on GC-MS to detect ZEN in aqueous environments. Zearalenone concentrations were determined at 0.3 to $0.5 \mathrm{ng} / \mathrm{L}$ in water samples, and recovery rates ranged from $62 \%$ to $80 \%$.

\subsection{Thin-layer chromatography}

Classical thin-layer chromatography (TLC) is a less accurate technique than GS-MS. Quantitative results are compared with the standard, and the purity, quality and concentration of the standards affect the accuracy of the analysis. This method has been used to detect ZEN in maize. The fluorescence of ZEN was compared against the applied standards. Samples that tested positive for ZEN in TLC were extracted again to validate the results [51].

Schaafsma et al. [57] relied on TLC to analyze Fusarium toxins in maize and wheat. A comparison of the costs associated with different analytical methods 
revealed that TLC was the cheapest technique. However, toxin concentrations were less accurately determined by TLC than HPLC.

\section{Conclusions}

The adverse effects of ZEN can contribute to many hormonedependent diseases. Zearalenone is ubiquitous in food products, which spurs the search for effective methods of neutralizing this toxin. Research into ZEN metabolism provides crucial information for evaluating this mycotoxin's effects in humans and animals. Quantitative analyses of ZEN in food and feed also play a very important role in such assessments. Effective analytical procedures are thus needed to minimize the negative impact of mycotoxins on living organisms and the environment. An attempt was made in this review article to present various methods for the isolation and quantification of ZEN and its metabolites. The optimal analytical method should be characterized by a simple sample preparation procedure because numerous analytical steps can lead to uncertainty in the accurate determination of the analytes. Several sample preparation techniques for different matrices were presented, and the applicability of various ZEN identification methods, mostly chromatography with different detection methods, was discussed.

Conflict of interest: Authors state no conflict of interest.

\section{References}

[1] Marin S, Ramos AJ, Cano-Sancho G, Sanchis V. Mycotoxins: occurrence, toxicology, and exposure assessment. Food Chem Toxicol. 2013;60:218-37.

[2] Buszewska-Forajta M. Mycotoxins, invisible danger of feedstuff with toxic effect on animals. Toxicon. 2020;182:34-53.Schelstraete W, Devreese M, Croubels S. Comparative toxicokinetics of Fusarium mycotoxins in pigs and humans. Food Chem Toxicol. 2020;137:111140.

[3] Urraca JL, Marazuela MD, Moreno-Bondi MC. Analysis for zearalenone and $\alpha$-zearalenol in cereals and swine feed using accelerated solvent extraction and liquid chromatography with fluorescence detection. Anal Chim Acta. 2004;524:175-83.

[4] Zinedine A, Soriano JM, Moltó JC, Mañes J. Review on the toxicity, occurrence, metabolism, detoxification, regulations and intake of zearalenone: an oestrogenic mycotoxin. Food Chem Toxicol. 2007;45(1):1-18.

[5] Rogowska A, Pomastowski P, Sagandykova G, Buszewski B. Zearalenone and its metabolites: effect on human health, metabolism and neutralisation methods. Toxicon. 2019;162(March):46-56

[6] Binder SB., Schwartz-Zimmermann HE, Varga E, Bichl G, Michlmayr $\mathrm{H}$, et al. Metabolism of zearalenone and its major modified forms in pigs. Toxins. 2017;9(2):1-15.
[7] Drzymala SS, Binder J, Brodehl A, Penkert M, Rosowski M, Garbe LA, et al. Estrogenicity of novel phase I and phase II metabolites of zearalenone and cis-zearalenone. Toxicon. 2015;105:10-2.

[8] Gadzała-Kopciuch R, Kwaśniewska K, Ludwiczak A, Skrzyniarz P, Jakubowski R, Nowak W, et al. Towards a new approach for the description of cyclo-2,4-dihydroxybenzoate, a substance which effectively mimics zearalenone in imprinted polymers designed for analyzing selected mycotoxins in urine. Int I Mol Sci. 2019;20:1588; doi:10.3390/ijms20071588.

[9] Metzler M. Proposal for a uniform designation of zearalenone and its metabolites. Mycotoxin Res. 2011;27(1):1-3.

[10] Bai X, Sun Ch, Xu J, Liu D, Han Y, Wu S, et al. Detoxification of zearalenone from corn oil by adsorption of functionalized $\mathrm{GO}$ systems. Appl Surf Sci. 2018;430:198-207.

[11] EFSA CONTAM Panel (EFSA Panel on Contaminants in the Food Chain). Scientific opinion on the risks to public health related to the presence of nickel in food and drinking water. EFSA J. 2015;13(2):1-124.

[12] Han Z, Jiang K, Fan Z, Di Mavungu JD, Dong M, Guo W, et al. Multi-walled carbon nanotubes-based magnetic solid-phase extraction for the determination of zearalenone and its derivatives in maize by ultra-high performance liquid chromatography-tandem mass spectrometry. Food Control. 2017;79:177-84.

[13] Olsen M, Pettersson H, Kiessling KH. Reduction of zearalenone to zearalenol in female rat liver by $3 \alpha-$ hydroxysteroid dehydrogenase. Acta Pharmacol Toxicol (Copenh). 1981;48(2):157-61.

[14] Ueberschär KH, Brezina U, Dänicke S. Zearalenone (ZEN) and ZEN metabolites in feed, urine and bile of sows: analysis, determination of the metabolic profile and evaluation of the binding forms. Landbauforsch Appl Agric Forestry Res. 2016;66(1):21-8.

[15] Gadzała-Kopciuch R, Cendrowski K, Cesarz A, Kietbasa $P$, Buszewski B. Determination of zearalenone and its metabolites in endometrial cancer by coupled separation techniques. Anal Bioanal Chem. 2011;401(7):2069-78.

[16] Pajewska M, Łojko M, Cendrowski K, Sawicki W, Kowalkowski T, Buszewski B, et al. The determination of zearalenone and its major metabolites in endometrial cancer tissues. Anal Bioanal Chem. 2018;410(5):1571-82.

[17] Zöllner P, Jodlbauer J, Lindner W. Determination of zearalenone in grains by high-performance liquid chromatography-tandem mass spectrometry after solid-phase extraction with RP-18 columns or immunoaffinity columns. J Chromatogr A. 1999;858(2):167-74.

[18] de Andrés F, Zougagh M, Castañeda G, Ríos A. Determination of zearalenone and its metabolites in urine samples by liquid chromatography with electrochemical detection using a carbon nanotube-modified electrode. J Chromatogr A. 2008;1212(1-2):54-60.

[19] Songsermsakul P, Sontag G, Cichna-Markl M, Zentek J, Razzazi-Fazeli E. Determination of zearalenone and its metabolites in urine, plasma and faeces of horses by HPLC-APCI-MS. J Chromatogr B Analyt Technol Biomed Life Sci. 2006;843(2):252-61.

[20] Mausia T, de Smet D, Guorun Q, van Peteghem C, Zhang $\mathrm{D}$, Wu A, et al. Molecularly imprinted polymers as specific adsorbents for zearalenone produced by precipitation 
polymerization and applied to mycotoxin production. Anal Lett. 2011;44(16):2633-43.

[21] Pallaroni L, Von Holst C. Development of an extraction method for the determination of zearalenone in corn using less organic solvents. J Chromatogr A. 2004; 1055(1-2):247-9.

[22] Biehl ML, Prelusky DB, Koritz GD, Hartin KE, Buck WB, Locksley Trenholm H. Biliary excretion and enterohepatic cycling of zearalenone in immature pigs. Toxicol Appl Pharmacol. 1993;121(1):152-9.

[23] Gambacorta L, Solfrizzo M, Visconti A, Powers S, Cossalter $A M$, Pinton $P$, et al. Validation study on urinary biomarkers of exposure for aflatoxin B1, ochratoxin A, fumonisin B1, deoxynivalenol and zearalenone in piglets. World Mycotoxin J. 2013;6(3):299-308.

[24] Dänicke S, Swiech E, Buraczewska L, Ueberschär KH. Kinetics and metabolism of zearalenone in young female pigs. J Anim Physiol Anim Nutr (Berl). 2005;89:268-76.

[25] Dänicke S, Brüssow KP, Valenta H, Ueberschär KH, Tiemann U, Schollenberger M. On the effects of graded levels of fusarium toxin contaminated wheat in diets for gilts on feed intake, growth performance and metabolism of deoxynivalenol and zearalenone. Mol Nutr Food Res. 2005;49:932-43.

[26] Warth B, Sulyok M, Berthiller F, Schuhmacher R, Krska R. New insights into the human metabolism of the Fusarium mycotoxins deoxynivalenol and zearalenone. Toxicol Lett. 2013;220(1):88-94.

[27] Ali N, Gisela HD. Urinary biomarkers of exposure to the mycoestrogen zearalenone and its modified forms in German Adults. Arch Toxicol. 2018;92(8):2691-700.

[28] Juan C, Rittieni A, Yusa V, Manes J. Determination of zearalenone and its metabolites in bovine feeding stuff and assessment residues in bovine urine. International Conference on Food Innovation (Food Innova'2010), 25-29 October 2010, Valencia, Spain. https://images.engormix. com/s_news/ZEARALENONE_ZERANOL(4).pdf.

[29] Woźny M, Obremski K, Jakimiuk E, Gusiatin M, Brzuzan P. Zearalenone contamination in rainbow trout farms in northeastern Poland. Aquaculture. 2013;416-417:209-11.

[30] Wu J, Zhao R, Chen B, Yang M. Determination of zearalenone in barley by high-performance liquid chromatography coupled with evaporative light scattering detection and natural occurrence of zearalenone in functional food. Food Chem. 2011;126(3):1508-11.

[31] De Baere S, Osselaere A, Devreese M, Vanhaecke L, De Backer $P$, Croubels S. Development of a liquid-chromatography tandem mass spectrometry and ultra-high-performance liquid chromatography high-resolution mass spectrometry method for the quantitative determination of zearalenone and its major metabolites in chicken and pig plasma. Anal Chim Acta. 2012;756:37-48.

[32] Hewitt TC, Flack CL, Kolodziejczyk JK, Chacon AM, D’Ovidio KL. Occurrence of zearalenone in fresh corn and corn products collected from local Hispanic markets in San Diego County, CA. Food Control. 2012;26(2):300-4.

[33] Jodlbauer J, Zöllner P, Lindner W. Determination of Zearalenone and its metabolites in urine and tissue samples of cow and pig by LC-MS/MS. Mycotoxin Res. 2000;16(2 SUPPL):174-8.

[34] Marczuk J, Obremski K, Lutnicki K, Gajecka M, Gajecki M. Zearalenone and deoxynivalenol mycotoxicosis in dairy cattle herds. Pol J Vet Sci. 2012;15(2):365-72.
[35] Ok HE, Jung H, Lee SE, Peak O, Chun HS. Three liquid chromatographic methods for the analysis of aflatoxins in for different corn (Zea Mays) matrices. J Food Compos Anal. 2016;54:20-6.

[36] Wozniak B, Matraszek-Zuchowska I, Zmudzki J. Determination of stilbenes and resorcylic acid lactones in bovine, porcine and poultry muscle tissue by liquid chromatography-negative ion electrospray mass spectrometry and QuEChERS for sample preparation. J Chromatogr B Analyt Technol Biomed Life Sci. 2013;940:15-23.

[37] Cunha SC, Fernandes JO. Development and validation of a method based on a QuEChERS procedure and heart-cutting GC-MS for determination of five mycotoxins in cereal products. J Sep Sci. 2010;33(4-5):600-9.

[38] Kim HJ, Kim SH, Lee JK, Choi CU, Lee HS, Kang HG, et al. A novel mycotoxin purification system using magnetic nanoparticles for the recovery of aflatoxin $\mathrm{Bl}$ and zearalenone from feed. J Vet Sci. 2012;13(4):363-9.

[39] Amoli-Diva M, Taherimaslak Z, Allahyari M, Pourghazid K. Dispersive liquid-liquid microextraction coupled with magnetic nanoparticles for extraction of zearalenone in wheat samples. Nanochem Res. 2017;2(1):60-70.

[40] Antep H. Mine, Merdivan M. Development for new dispersive liquid-liquid microextraction technique for the identification of zearalenone in beer. Anal Methods. 2012;4(12):4129-34.

[41] Hashemi M, Taherimaslak Z, Parvizi S, Torkejokar M. Spectrofluorimetric Determination of zearalenone using dispersive liquid-liquid microextraction coupled to micro-solid phase extraction onto magnetic nanoparticles. RSC Advances. 2014;4(85):45065-73.

[42] Porto-Figueira P, Camacho I, Câmara JS. Exploring the potentialities of an improved ultrasound-assisted quick, easy, cheap, effective, rugged, and safe-based extraction technique combined with ultrahigh pressure liquid chromatographyfluorescence detection for determination of zearalenone in cereals. J Chromatogr A. 2015;1408:187-96.

[43] Cavaliere C, Antonelli M, Cerrato A, La Barbera G, Laganà A, Laus $M$, et al. A novel magnetic molecular imprinted polymer for selective extraction of zearalenone from cereal flours before liquid chromatography-tandem mass spectrometry determination. Toxins (Basel). 2019;11(9):1-12.

[44] Fang G, Fan C, Liu H, Pan M, Zhu H, Wang S. A novel molecularly imprinted polymer on $\mathrm{CdSe} / \mathrm{ZnS}$ quantum dots for highly selective optosensing of mycotoxin zearalenone in cereal samples. RSC Advances. 2014;4(6):2764-71.

[45] Sforza S, Dall'asta C, Marchelli R. Recent advances in mycotoxin determination in food and feed by hyphenated chromatographic techniques/mass spectrometry. Mass Spectrom Rev. 2006;25(1):54-76.

[46] Soleimany F, Jinap S, Faridah A, Khatib AA. UPLC-MS/MS for simultaneous determination of aflatoxins, ochratoxin $A$, zearalenone, DON, fumonisins, T-2 toxin and HT-2 toxin, in cereals. Food Control. 2012;25(2):647-53.

[47] National Institute for Public Health and the Environment. Analysis of zeranol and metabolites in urine, bile, meat, liver and kidney by GC-MS. Standard Operating Procedure: ARO/458;1-9; https://www.rivm.nl/bibliotheek/ digitaaldepot/ARO458EN.pdf. 
[48] Rodríguez-Carrasco Y, Moltó JC, Berrada H, Mañes J. A survey of trichothecenes, zearalenone and patulin in milled grain-based products using GC-MS/MS. Food Chem. 2014;146:212-9.

[49] Dudziak M. Analysis of zearalenone in aqueous environment using GC-MS. Pol J Environ Stud. 2011;20(1):231-5.

[50] Shotwell OL, Hesseltine CE, Goulden ML, Vandegraft EE. Survey of corn for aflatoxin, zearalenone, and ochratoxin. Cereal Chem. 1970;4(6):700-7.

[51] Duca RC, Bravin F, Delaforge M, Vladescu L, Badea IA, Criste RD. Development of a new HPLC method used for determination of zearalenone and its metabolites in broiler samples. Influence of zearalenone on the nutritional properties of broiler meat. J Agric Food Chem. 2009;57(22):10497-504.

[52] Llorent-Martínez EJ, Fernández-Poyatos MP, Ruiz-Medina A. Automated fluorimetric sensor for the determination of zearalenone mycotoxin in maize and cereals feedstuff. Talanta. 2019;191:89-93.
[53] Vendl O, Berthiller F, Crews C, Krska R. Simultaneous determination of deoxynivalenol, zearalenone, and their major masked metabolites in cereal-based food by LC-MS-MS. Anal Bioanal Chem. 2009;395(5):1347-54.

[54] Kinani S, Bouchonnet S, Bourcier S, Porcher JM, Aït-Aïssa S. Study of the chemical derivatization of zearalenone and its metabolites for gas chromatography-mass spectrometry analysis of environmental samples. J Chromatogr A. 2008;1190(1-2):307-15.

[55] Blokland MH, Sterk SS, Stephany RW, Launay FM, Kennedy DG, van Ginkel LA. Determination of resorcylic acid lactones in biological samples by GC-MS. Discrimination between illegal use and contamination with fusarium toxins. Anal Bioanal Chem. 2006;384(5):1221-7.

[56] Schaafsma AW, Nicol RW, Savard ME, Sinha RC, Reid LM, Rottinghaus G. Analysis of Fusarium toxins in maize and wheat using thin layer chromatography. Mycopathologia. 1998;142(2):107-13. 